\title{
Assessment of treatment approach and survival outcomes in a modern cohort of patients with primary gliosarcoma using the national cancer database
}

\author{
Lu Deng ${ }^{1}$, Allison Cheung ${ }^{2}$, Harrison X. $\mathrm{Bai}^{3}$, Xuejun $\mathrm{Li}^{4}$, Weihua Liao ${ }^{5}, \mathrm{Ya} \mathrm{Cao}^{6}$, \\ Giorgos Karakousis ${ }^{7}$, Paul J. Zhang ${ }^{8}$, Bo Xiao ${ }^{9}$ and Li Yang ${ }^{1}$ \\ ${ }^{1}$ Department of Neurology, The Second Xiangya Hospital, Central South University, Changsha, Hunan, 410011, China \\ ${ }^{2}$ Department of Molecular, Cellular and Developmental Biology, Yale University, New Haven, Connecticut, 06511, United States \\ ${ }^{3}$ Department of Radiology, Hospital of the University of Pennsylvania, Philadelphia, Pennsylvania, 19104, United States \\ ${ }^{4}$ Department of Neurosurgery, Xiangya Hospital, Central South University, Changsha, Hunan, 410008, China \\ ${ }^{5}$ Department of Radiology, Xiangya Hospital, Central South University, Changsha, Hunan, 410008, China \\ ${ }^{6}$ Cancer Research Institute, School of Basic Medicine, Central South University, Changsha, Hunan, 410078, China \\ ${ }^{7}$ Department of Surgery, Hospital of the University of Pennsylvania, Silverstein, Philadelphia, Pennsylvania, 19104, United States \\ ${ }^{8}$ Department of Pathology and Laboratory Medicine, Hospital of the University of Pennsylvania, Philadelphia, Pennsylvania, 19104, \\ United States \\ ${ }^{9}$ Department of Neurology, Xiangya Hospital, Central South University, Changsha, Hunan, 410008, China \\ Correspondence to: Li Yang, email: yangli762@csu.edu.cn \\ Bo Xiao, email: Xiaobo_xy@126.com
}

Keywords: age, gliosarcoma, national cancer database, survival, trimodality therapy

Received: April 11, 2017 Accepted: August 26, $2017 \quad$ Published: January 12, 2018

Copyright: Deng et al. This is an open-access article distributed under the terms of the Creative Commons Attribution License 3.0 (CC BY 3.0 ), which permits unrestricted use, distribution, and reproduction in any medium, provided the original author and source are credited.

\section{ABSTRACT}

Background: Primary gliosarcoma is a rare malignant brain tumor with dismal prognosis. Previous reports are limited to case reports and small retrospective case series.

Objective: To evaluate treatment and survival outcomes in a large cohort of primary gliosarcoma patients treated in the United States.

Results: 1622 patients met the inclusion criterion. Median age was 63 years. The 3-year OS rate for the entire cohort was $11.9 \%$. Patients aged 18 to 60 years were significantly more likely to receive trimodality therapy (defined as the use of surgery, radiotherapy $[\mathrm{RT}]$ and chemotherapy $[\mathrm{CT}])$ than patients older than $60(68.1 \% \mathrm{vs}$. $56.7 \%, p<0.001)$. The utilization of trimodality therapy significantly increased during the study period $(57.5 \%$ in $2004-2008$ vs. $65.1 \%$ in $2009-2013 ; p=0.002)$. On multivariate Cox regression analysis, GTR, surgery followed by RT and the use of trimodality therapy were associated with longer OS, while older age, Charlson-Deyo score $\geq 1$ and multi-focal tumor were associated with shorter OS. The use of trimodality therapy was consistently associated with longer OS in subgroup analyses based on age and extent of resection.

Materials and Methods: The National Cancer Database was used to identify all primary gliosarcoma patients aged 18 to 90 years who were diagnosed between 2004 and 2013. Overall survival (OS) was evaluated by Kaplan-Meir analysis, univariate and multivariate Cox proportional hazard regression analysis.

Conclusions: The use of trimodality therapy significantly increased during the study period and was associated with improved outcomes regardless of age and extent of resection. 


\section{INTRODUCTION}

Gliosarcoma (GSM) is a rare, primary malignant brain tumor that accounts for $4 \%$ of all malignant gliomas [1]. Its morphology is characterized as a wellcircumscribed lesion with clearly identifiable biphasic glial and mesenchymal components histologically $[1,2]$. The mesenchymal component of the tumor may display a variety of morphologies with origins from fibroblastic, cartilaginous, osseous, smooth muscle, striated muscle, or adipose cell lineage [3, 4]. Clinical guidelines for diagnosis are poorly defined. In the 2007 World Health Organization (WHO) classification of central nervous system (CNS) tumors, the glial component of GSM meets the histological criteria for grade IV astrocytoma (glioblastoma) [5]. The new 2016 WHO classification, with its incorporation of molecular markers, has placed GSM under the category of IDH-wild type glioblastoma [6].

Clinically similar to glioblastoma, GSM usually affects patients in their sixth to eighth decade of life, with a male preponderance $[7,8]$. The median age at diagnosis was approximately 60 years [7, 9-12].Current treatment options include surgery, radiotherapy (RT) and chemotherapy (CT) [13]. Due to its low incidence, the current literature is limited to case reports and small cases series with insufficient evidence to guide treatment decisions. A previous study using the National Cancer Data Base (NCDB) compared patient demographics, treatment regimen, and survival among glioblastoma, giant cell glioblastoma, and GSM [14]. However, the study was limited to patients diagnosed before 2006 and did not provide focused analysis on the GSM patients.

The primary aim of this study was to examine treatment approach and survival outcomes in patients with primary GSM using data from the NCDB.

\section{RESULTS}

\section{Demographic, patient, and tumor characteristics}

A total of 1622 patients were included with a median follow-up of 10.2 months. Our patient flowchart is shown in Supplementary Figure 1. The median age was 63 years (range, 18-90 years). A summary of patient and tumor characteristics for the entire cohort is shown in Table 1. Information on the extent of resection was available for 524 patients, among whom 309 underwent gross total resection (GTR) and 215 subtotal resection (STR) (Supplementary Table 1). A comparison of baseline characteristics of patients diagnosed before and after 2007 is shown in Supplementary Table 2.

\section{Treatment}

One thousand patients $(61.7 \%)$ received trimodality therapy (defined as the use of surgery, RT and CT), while
365 patients received surgery alone, 164 patients surgery followed by RT, and 30 patients surgery followed by CT. Sixty-three patients did not undergo surgery. Patients aged 18 to 60 years were significantly more likely to receive trimodality therapy than patients older than $60(68.1 \%$ vs. $56.7 \%, p<0.001)$. The use of trimodality therapy significantly increased during the study period $(57.5 \%$ in $2004-2008$ vs. $65.1 \%$ in $2009-2013 ; p=0.002$ ). Patients aged $18-60$ years were significantly more likely to receive RT $(77.0 \%$ vs. $67.7 \%, p<0.001)$ and CT $(69.2 \%$ vs. $59.1 \%$, $p<0.001)$ than patients aged $61-90$ years. The use of RT (69.4\% for $2004-2008$ vs. $73.7 \%$ for $2009-2013, p=0.06$ ) and CT $(59.9 \%$ in $2004-2008$ vs. $65.5 \%$ in $2009-2013$, $p=0.007$ ) increased significantly during the study period.

\section{Survival outcomes}

The median OS and the 3-year OS rate for the entire cohort was 10.2 months and $11.9 \%$, respectively (Figure 1). Patients diagnosed after 2007 had significant longer OS than those diagnosed before 2007 (Supplementary Figure 2). On multivariate analysis, older age (HR, 1.032, 95\% CI, 1.028-1.037, $p<0.001$ ), Charlson-Deyo score $\geq 1$ (HR, 1.262, 95\% CI, 1.125 $1.416, p<0.001)$ and multi-focal tumor (HR, 1.417, $95 \%$ CI, 1.105-1.817, $p=0.006)$ were associated with shorter OS, while GTR (HR, 0.784, 95\% CI, 0.659-0.933, $p=0.006$ ), surgery followed by RT (HR, $0.783,95 \%$ CI, $0.645-0.950, p=0.01$ ) and the use of trimodality therapy (HR, $0.514,95 \%$ CI, $0.451-0.585, p<0.001$ ) were associated with longer OS (Table 2 and Figure 2). Compared to surgery alone, surgery followed by $\mathrm{CT}$ alone did not result in improved OS on multivariate analysis.

\section{Subgroup analysis based on extent of resection}

There was no significant difference in baseline characteristics between STR and GTR groups except for tumor size (Supplementary Table 1). Patients who received GTR had significantly longer OS than those who received STR (median OS: 13.2 vs. 9.6 months, $p<0.001$; Figure 2B). On multivariate analysis, among the 215 patients who underwent STR, older age (HR, $1.021,95 \% \mathrm{CI}, 1.008-1.034, p=0.002)$ and multi-focal tumor (HR, 1.912, 95\% CI, 1.236-2.958, $p=0.004$ ) were associated with shorter OS, while the use of trimodality therapy was associated with longer OS (HR, $0.375,95 \%$ CI, 0.259-0.543, $p<0.001$ ) (Supplementary Table 3 and Supplementary Figure 3).

Among the 309 patients who underwent GTR, older age (HR, 1.029, 95\% CI, 1.017-1.041, $p<0.001$ ) and a Charlson-Deyo score > 1 (HR, 1.391, 95\% CI, 1.044-1.854, $p=0.02$ ) were associated with shorter OS, while the use of trimodality therapy was associated with longer OS (HR, 0.545 , $95 \%$ CI, 0.388-0.764, $p<0.001)$ on multivariate analysis (Supplementary Table 4 and Supplementary Figure 4). 
Table 1: Demographics and clinical characteristics of the entire cohort

\begin{tabular}{|c|c|c|}
\hline Category & & No. $(\%)$ \\
\hline \multirow[t]{5}{*}{ Age (range, $18-90$ y) } & $18-40 y$ & $102(6.3)$ \\
\hline & $41-50 y$ & $203(12.5)$ \\
\hline & $51-60 y$ & $400(24.7)$ \\
\hline & $61-70 y$ & $484(29.8)$ \\
\hline & $71-90$ y & $433(26.7)$ \\
\hline \multirow[t]{2}{*}{ Gender } & Male & $987(60.9)$ \\
\hline & Female & $635(39.1)$ \\
\hline \multirow[t]{5}{*}{ Race } & White & $1426(87.9)$ \\
\hline & Black & $133(8.2)$ \\
\hline & Asian & $31(1.9)$ \\
\hline & Other & $13(0.8)$ \\
\hline & Unknown & $19(1.2)$ \\
\hline \multirow[t]{2}{*}{ Year of diagnosis } & 2004-2008 & 739 (45.6) \\
\hline & 2009-2013 & $883(54.4)$ \\
\hline \multirow[t]{3}{*}{ Tumor size } & $0-4 \mathrm{~cm}$ & $502(30.9)$ \\
\hline & $>4.1 \mathrm{~cm}$ & $818(50.4)$ \\
\hline & Unknown & $302(18.6)$ \\
\hline \multirow[t]{3}{*}{ Tumor location } & Supratentorial & $1282(79.0)$ \\
\hline & Infratentorial & $10(0.6)$ \\
\hline & Unknown & $330(20.3)$ \\
\hline \multirow[t]{3}{*}{ Metastasis at diagnosis } & M0 & $1486(91.6)$ \\
\hline & $\mathrm{M}+$ & $21(1.3)$ \\
\hline & Unknown & $115(7.1)$ \\
\hline \multirow[t]{2}{*}{ Charlson-Deyo score } & 0 & $1140(70.3)$ \\
\hline & $\geq 1$ & $482(29.7)$ \\
\hline \multirow[t]{5}{*}{ Treatment } & No surgery & $63(3.9)$ \\
\hline & Surgery alone & $365(22.5)$ \\
\hline & Surgery with RT & $164(10.1)$ \\
\hline & Surgery with CT & $30(1.8)$ \\
\hline & Trimodality therapy & $1000(61.7)$ \\
\hline \multirow[t]{3}{*}{ Extent of Resection } & Subtotal resection/biopsy & $344(21.2)$ \\
\hline & Gross total resection & $309(19.1)$ \\
\hline & Other & $969(59.7)$ \\
\hline \multirow[t]{3}{*}{ Lesion Number } & Unifocal tumor & $568(35.0)$ \\
\hline & Multifocal tumor & $83(5.1)$ \\
\hline & Other & $971(59.9)$ \\
\hline
\end{tabular}

Abbreviations: M0, no metastatic disease at diagnosis; M+, metastatic disease at diagnosis; RT, radiation therapy; CT, chemotherapy.

\section{Subgroup analysis based on age}

The age distribution of the entire cohort is shown in Supplementary Figure 5. There was no significant difference in baseline characteristics between the 18-60 y and 61-90 y age groups except for race, Charlson-Deyo score and treatment (Supplementary Table 5). Older patients were more likely to be white and with more comorbidities, but less likely to receive trimodality than younger patients. In the 18-60 age group, older age (HR, $1.030,95 \%$ CI, $1.019-1.040, p<0.001)$, a Charlson-Deyo score $>1$ (HR, 1.219, 95\% CI, 1.002-1.482, $p=0.047)$ 
and multi-focal tumor (HR, 1.747, 95\% CI, 1.173-2.601, $p=0.006)$ were associated with shorter OS, while trimodality therapy (HR, 0.599, 95\% CI, 0.481-0.745, $p<0.001$ ) and GTR (HR, 0.761, 95\% CI, 0.580-0.999, $p=0.049$ ) were associated with longer OS on multivariate analysis (Supplementary Table 6 and Supplementary Figure 6).

In the 61-90y group, trimodality therapy (HR, $0.469,95 \%$ CI, 0.397-0.554, $p<0.001)$ was associated with longer OS, while older age (HR, 1.045, 95\% CI, 1.034-1.055, $p<0.001)$ and a Charlson-Deyo score $>$ 1 (HR, 1.316, 95\% CI, 1.138-1.522, $p<0.001$ ) were associated with shorter OS (Supplementary Table 7 and Supplementary Figure 7).

\section{DISCUSSION}

Due to the rarity of GSM, insufficient literature has been published on this tumor to guide clinical treatment [15]. The current treatment recommendations for GSM are largely based on those for the more common glioblastoma. Using the NCDB, we evaluated treatment approaches and survival outcomes in 1622 adult patients diagnosed with primary GSM. We found that the use of trimodality therapy significantly increased during the study period and was associated with longer OS. Compared to surgery alone, surgery followed by RT alone also resulted in improved OS, but not to the same extent as trimodality therapy. Surgery followed by CT alone did not result in an OS benefit. These results were consistent in subgroup analyses as well.

The improved survival during the study period could be attributed to the increased use of trimodality therapy. We found that the use of both RT $(p=0.06)$ and CT $(p=0.007)$ increased significantly during the time period. These changes could be attributed to the improved understanding of underlying tumor biology as well as tumor response to these adjuvant therapies in recent years [16-18], or more importantly the clinical findings in both GSM and the clinically similar glioblastoma that more favorable outcomes often result from treatment with adjuvant RT and/or CT [19, 20]. This same trend has started to apply to low-grade gliomas as well, after a randomized controlled trial of 251 patients was published in 2016 that demonstrated longer progression-free survival and OS among patients who received trimodality when compared to RT alone [21]. However, there are wellknown side effects of RT and CT which are especially prominent for elderly cancer patients treated with these therapies $[22,23]$. Thus, these treatments should only be offered when the benefits outweigh the risks.

A number of studies on glioblastomas have demonstrated the efficacy of RT and CT, in addition to surgery, as the treatment of choice [19, 24, 25]. The question that remains, which was investigated in our study, is whether or not this standard of treatment can be applied to GSM patients, given that GSM has a worse prognosis than glioblastoma $[20,25]$. In our cohort of GSM patients, we found that although surgery followed by RT alone was associated with longer OS, trimodality therapy resulted in the greatest OS benefit. Despite the increasing use of RT and $\mathrm{CT}$ in recent years, the current literature has mixed results on the effectiveness of adjuvant therapy in GSM. Some studies have demonstrated the benefit of adjuvant RT and CT $[25,26]$, while others questioned their utility $[25,26]$. A literature summary of treatment, survival and prognostic factors in GSM studies greater than 10 patients is presented in Supplementary Table $8[2,7-12$, $20,24-35]$. In many of these studies, the sample size was small, and there was a lot of heterogeneity in the treatment regimen used. In a study of 353 GSM patients from the Surveillance, Epidemiology, and End Results database, the largest study published in the literature prior to the current study, Kozak et al. suggested that tumor resection, as opposed to biopsy only, and adjuvant RT may improve

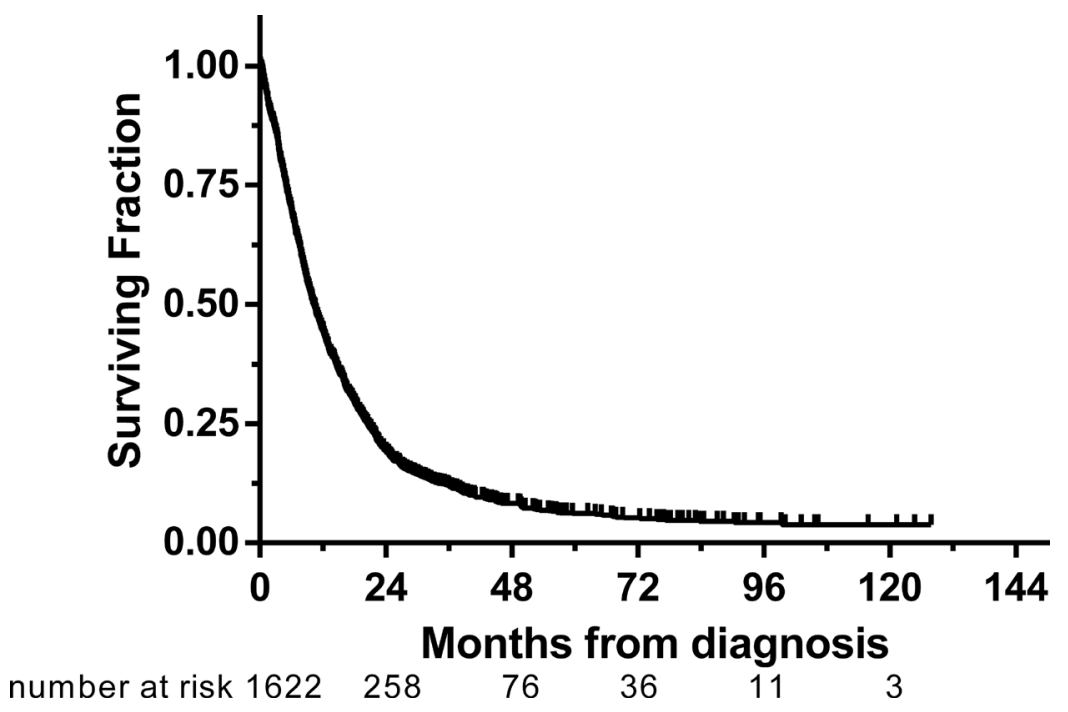

Figure 1: Kaplan-Meier curve of overall survival for the entire cohort. 
Table 2: Univariate and multivariate Cox proportional hazards analyses of the overall survival for the entire cohort

\begin{tabular}{|c|c|c|c|c|}
\hline \multirow[b]{2}{*}{ Variable } & \multicolumn{2}{|c|}{ Univariate Analysis } & \multicolumn{2}{|c|}{ Multivariate Analysis } \\
\hline & HR $(95 \%$ CI $)$ & $p$ & HR $(95 \%$ CI) & $p$ \\
\hline \multicolumn{5}{|l|}{ Age (range, 18-90 y) } \\
\hline As continuous variable & $1.035(1.030-1.039)$ & $<0.001$ & $1.032(1.028-1.037)$ & $<0.001$ \\
\hline \multicolumn{5}{|l|}{ Gender } \\
\hline Male & Reference group & & - & \\
\hline Female & $0.934(0.840-1.040)$ & 0.21 & - & \\
\hline \multicolumn{5}{|l|}{ Race } \\
\hline White & Reference group & & - & \\
\hline Nonwhite & $0.873(0.742-1.027)$ & 0.10 & - & \\
\hline \multicolumn{5}{|l|}{ Year of diagnosis } \\
\hline 2004-2008 & Reference group & & - & \\
\hline 2009-2013 & $0.890(0.801-0.988)$ & 0.03 & - & \\
\hline \multicolumn{5}{|l|}{ Tumor size } \\
\hline $0-4.0 \mathrm{~cm}$ & Reference group & & - & \\
\hline$>4.0 \mathrm{~cm}$ & $1.064(0.945-1.199)$ & 0.30 & - & \\
\hline \multicolumn{5}{|l|}{ Tumor location } \\
\hline Supratentorial & Reference group & & - & \\
\hline Infratentorial & $0.574(0.273-1.207)$ & 0.14 & - & \\
\hline \multicolumn{5}{|l|}{ Metastasis at diagnosis } \\
\hline M0 & Reference group & & - & \\
\hline $\mathrm{M}+$ & $1.074(0.674-1.710)$ & 0.76 & - & \\
\hline \multicolumn{5}{|l|}{ Charlson-Deyo score } \\
\hline 0 & Reference group & & Reference group & \\
\hline$\geq 1$ & $1.398(1.248-1.566)$ & $<0.001$ & $1.262(1.125-1.416)$ & $<0.001$ \\
\hline \multicolumn{5}{|l|}{ Treatment } \\
\hline Surgery alone & Reference group & & Reference group & \\
\hline No surgery & $1.096(0.827-1.452)$ & 0.52 & $1.136(0.851-1.518)$ & 0.39 \\
\hline Surgery with RT & $0.849(0.700-1.031)$ & 0.10 & $0.783(0.645-0.950)$ & 0.01 \\
\hline Surgery with CT & $1.036(0.704-1.525)$ & 0.86 & $0.895(0.607-1.318)$ & 0.57 \\
\hline Trimodality therapy & $0.507(0.446-0.577)$ & $<0.001$ & $0.514(0.451-0.585)$ & $<0.001$ \\
\hline \multicolumn{5}{|l|}{ Extent of resection } \\
\hline Subtotal resection/Biopsy & Reference group & & Reference group & \\
\hline Gross total resection & $0.722(0.608-0.858)$ & $<0.001$ & $0.784(0.659-0.933)$ & 0.006 \\
\hline Other & $0.959(0.839-1.095)$ & 0.53 & $1.028(0.806-1.311)$ & 0.82 \\
\hline \multicolumn{5}{|l|}{ Lesion number } \\
\hline Unifocal tumor & Reference group & & Reference group & \\
\hline Multifocal tumor & $1.461(1.141-1.872)$ & 0.003 & $1.417(1.105-1.817)$ & 0.006 \\
\hline Other & $1.136(1.014-1.272)$ & 0.03 & $0.992(0.783-1.256)$ & 0.95 \\
\hline
\end{tabular}

Abbreviations: CI, confidence interval; HR, hazard ratio; M0, no metastatic disease at diagnosis; $\mathrm{M}+$, metastatic disease at diagnosis; RT, radiation therapy; CT, chemotherapy. 
survival outcomes [20]. However, this study was limited by the lack of data on CT. In a retrospective study of 75 GSM patients by Castelli et al., the second-largest of its kind, patients were treated with a combination of surgery, RT and/or CT. A high total dose of RT and treatment at recurrence were found to be good prognostic factors, while temozolomide (TMZ)-based CT was not associated with an improvement in OS when compared to patients who received RT alone [9]. A smaller retrospective study of 27 GSM patients from 2009-2013 by Kumar et al. found that the addition of TMZ to RT did not significantly improve OS when compared to RT alone [33]. Using a much larger cohort, we were able to show definitively the benefit of trimodality therapy in both the entire cohort and subgroups of GTR and STR.

Age has been shown to be an important predictor of survival in both GSM and glioblastoma. We found that patients over the age of 60 years were less likely to
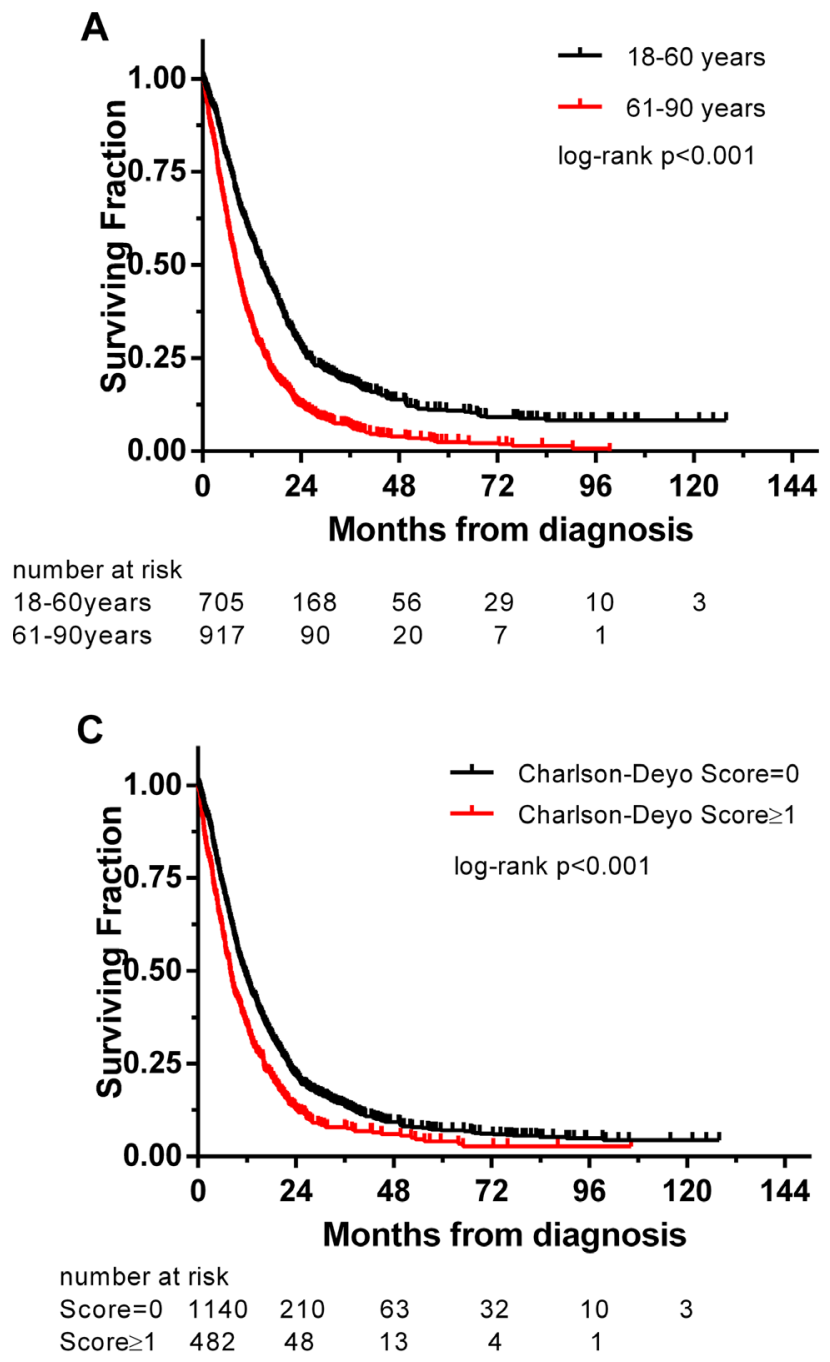

receive adjuvant therapies than younger patinets. Similar trends have been observed in glioblastoma patients, in which age was the most significant predictor of the type of treatment received, with elderly patients less likely to receive adjuvant $\mathrm{RT}$ and $\mathrm{CT}[36,37]$. As expected, the survival outcomes for younger patients were significantly better than those for older patients (median OS: $14.5 \mathrm{vs}$. 8.2 months; $p<0.001)$. In addition to the less favorable tumor biology and comorbidities associated with advanced age, it is possible that the shorter OS in older patients can be partly attributed to their lesser likelihood of receiving trimodality therapy, which has been shown in our study to improve OS regardless of age. The concern about adjuvant RT in elderly glioblastoma patients is neurotoxicity, which would outweigh any survival benefit conferred by RT [38-40]. The link between comorbidity and receipt of treatment remains unclear, even in glioblastoma patients. For example, Iwamoto et al. found that though higher

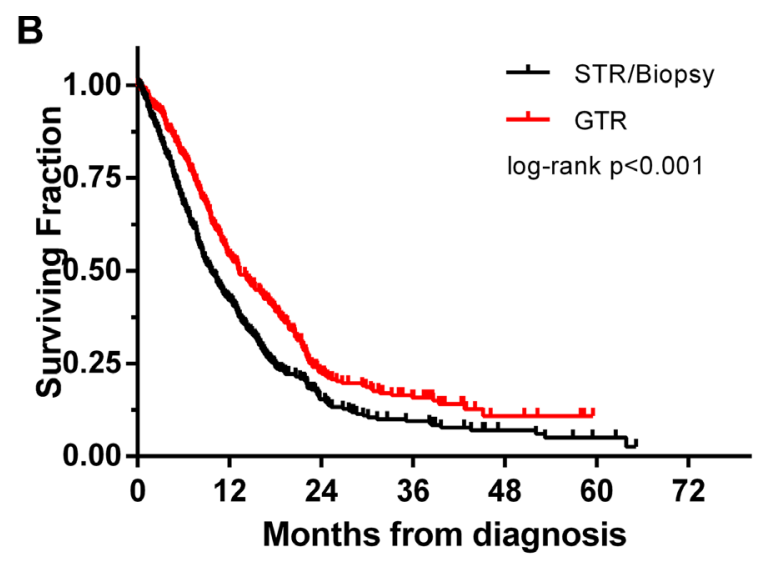

number at risk

$\begin{array}{lrrrrrr}\text { STR/Biopsy } & 344 & 133 & 36 & 18 & 7 & 3 \\ \text { GTR } & 309 & 157 & 47 & 26 & 5 & \end{array}$

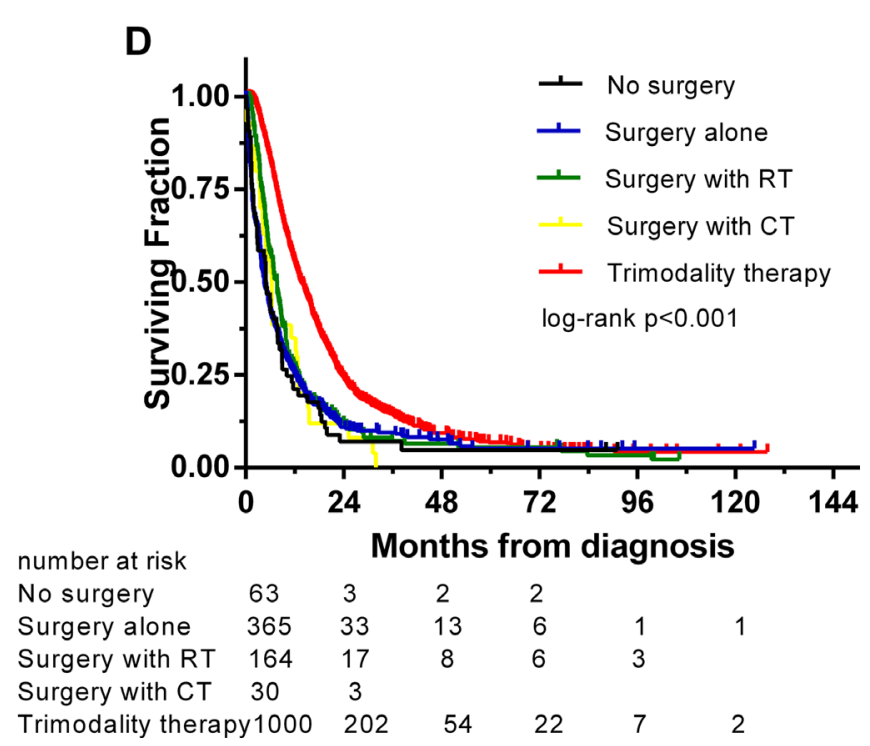

Figure 2: Kaplan-Meier curve comparing overall survival in patients (A) aged 18 to 60 years and 61 to 90 years, (B) who underwent subtotal resection/biopsy and gross total resection, (C) with Charlson-Deyo score of 0 and $\geq 1$, (D) treated with no surgery, surgery alone, surgery followed by radiation therapy, surgery followed by chemotherapy and trimodality therapy. 
comorbidity was associated with decreased RT treatment, comorbidities were not strong predictors of treatment receipt [41].

We would like to acknowledge several limitations of our study. First, there was no central pathology review so presumably some samples may have been misdiagnosed as GSM. Second, data on rescue therapies post primary course of treatment was not available in the NCDB. Third, the NCDB lacked data on important molecular markers in glioblastoma such as IDH1 mutation and MGMT promoter methylation. However, IDH1 mutation and MGMT promoter methylation have been reported to be rare in GSM [2]. Even though our study includes a large number of patients with relatively complete information on patient demographics and treatments, the inherent bias of a retrospective study can only be excluded with a prospective randomized control trial.

\section{MATERIALS AND METHODS}

\section{Data source and study population}

The NCDB is a joint project of the Commission on Cancer of the American College of Surgeons and the American Cancer Society. Established in 1989, the NCDB is a comprehensive, nationwide, facility-based oncology data set that captures approximately $70 \%$ of all newly diagnosed malignancies in the United States. The data used in this study are derived from a deidentified participant user file. The American College of Surgeons and the Commission on Cancer have not verified and are not responsible for the analytic or statistical methodology used, or for the conclusions drawn, from these data by the investigators.

Deidentified data for patients diagnosed with primary GSM who were 18 years or older and diagnosed between 2004 and 2013 with follow-up were extracted from the NCDB. We excluded patients with incomplete treatment data. Demographic and clinical data extracted included age, gender, race, primary tumor site, tumor size, presence of metastatic disease, Charlson-Deyo score, extent of resection (GTR versus STR/biopsy), lesion number (unifocal versus multifocal) and type of treatment (surgery, RT, and CT). Neither institutional review board/ethics committee nor patient consent was required for this study since the study used de-identified data from a public database.

\section{Statistical analysis}

OS was calculated from diagnosis until death with censoring at the last follow-up for patients who were alive. Patients' co-morbidity, or the lack of, was evaluated by the Charlson-Deyo score. Charlson-Deyo score $(0,1$, or 2) was assigned according to NCDB guidelines based on how many co-morbid conditions were reported and their relative severity. The chi-square test was used to evaluate contingency tables as appropriate. Univariate
Cox regression followed by multivariate Cox proportional hazard regression was used to calculate hazard ratios (HRs) for survival and identify independent prognostic factors for OS. Variables with $p$ values $<0.05$ on univariate cox regression were entered into the multivariate Cox proportional model. Significance was defined as a value of $p<0.05$. All levels of significance were 2-sided. SPSS Statistics V22.0 (SPSS Inc., Chicago, IL) was used for all statistical analyses.

\section{CONCLUSIONS}

In conclusion, the use of trimodality therapy significantly increased during the study period and was associated with longer survival regardless of age or extent of resection.

\section{Abbreviations}

NCDB, National Cancer Database; OS, overall survival; CI, confidence interval; GSM, gliosarcoma; CNS, central nervous system; WHO, World Health Organization; RT, radiotherapy; CT, chemotherapy; GTR, gross total resection; STR, subtotal resection; HR, hazard ratio; TMZ, temozolomide.

\section{Author contributions}

Li Yang, Harrison X. Bai and Lu Deng conceptualized the project. Harrison X. Bai obtained the data used for this study. All authors contributed to data analysis and editing of the manuscript.

\section{CONFLICTS OF INTEREST}

The authors have declared that no competing interests exist.

\section{FUNDING}

This study was supported by Shenghua Yuying Project of Central South University to L.Y., National Science Foundation of China to XJL (81472594) and National Science Foundation of China to WHL (81671676).

\section{REFERENCES}

1. Karsy M, Gelbman M, Shah P, Balumbu O, Moy F, Arslan E. Established and emerging variants of glioblastoma multiforme: review of morphological and molecular features. Folia Neuropathol. 2012; 50:301-321.

2. Lee D, Kang SY, Suh YL, Jeong JY, Lee JI, Nam DH. Clinicopathologic and genomic features of gliosarcomas. J Neurooncol. 2012; 107:643-650. 
3. Reis RM, Konu-Lebleblicioglu D, Lopes JM, Kleihues P, Ohgaki H. Genetic profile of gliosarcomas. Am J Pathol. 2000; 156:425-432.

4. Biernat W, Aguzzi A, Sure U, Grant JW, Kleihues P, Hegi ME. Identical mutations of the p53 tumor suppressor gene in the gliomatous and the sarcomatous components of gliosarcomas suggest a common origin from glial cells. J Neuropathol Exp Neurol. 1995; 54:651-656.

5. Louis DN, Ohgaki H, Wiestler OD, Cavenee WK, Burger PC, Jouvet A, Scheithauer BW, Kleihues P. The 2007 WHO classification of tumours of the central nervous system. Acta Neuropathol. 2007; 114:97-109.

6. Louis DN, Perry A, Reifenberger G, von Deimling A, Figarella-Branger D, Cavenee WK, Ohgaki H, Wiestler OD, Kleihues P, Ellison DW. The 2016 World Health Organization Classification of Tumors of the Central Nervous System: a summary. Acta Neuropathol. 2016; 131:803-820.

7. Han SJ, Yang I, Ahn BJ, Otero JJ, Tihan T, McDermott MW, Berger MS, Prados MD, Parsa AT. Clinical characteristics and outcomes for a modern series of primary gliosarcoma patients. Cancer. 2010; 116:1358-1366.

8. Lutterbach J, Guttenberger R, Pagenstecher A. Gliosarcoma: a clinical study. Radiother Oncol. 2001; 61:57-64.

9. Castelli J, Feuvret L, Haoming QC, Biau J, Jouglar E, Berger A, Truc G, Gutierrez FL, Morandi X, Le Reste PJ, Thillays F, Loussouarn D, Nouhaud E, et al. Prognostic and therapeutic factors of gliosarcoma from a multi-institutional series. J Neurooncol. 2016; 129:85-92.

10. Galanis E, Buckner JC, Dinapoli RP, Scheithauer BW, Jenkins RB, Wang CH, O'Fallon JR, Farr GJ. Clinical outcome of gliosarcoma compared with glioblastoma multiforme: North Central Cancer Treatment Group results. J Neurosurg. 1998; 89:425-430.

11. Damodaran O, van Heerden J, Nowak AK, Bynevelt M, McDonald K, Marsh J, Lee G. Clinical management and survival outcomes of gliosarcomas in the era of multimodality therapy. J Clin Neurosci. 2014; 21:478-481.

12. Adeberg S, Bernhardt D, Harrabi SB, Diehl C, Koelsche C, Rieken S, Unterberg A, von Deimling A, Debus J. Radiotherapy plus concomitant temozolomide in primary gliosarcoma. J Neurooncol. 2016; 128:341-348.

13. McAleer MF, Brown PD. Therapeutic management of gliosarcoma in the temozolomide era. CNS Oncol. 2015; 4:171-178.

14. Ortega A, Nuno M, Walia S, Mukherjee D, Black KL, Patil CG. Treatment and survival of patients harboring histological variants of glioblastoma. J Clin Neurosci. 2014; 21:1709-1713.

15. Yang L, Su C, Lee AM, Bai HX. Focusing on rare diseases in China: are we there yet? Orphanet J Rare Dis. 2015; 10:142.

16. Liu W, Lv G, Li Y, Li L, Wang B. Downregulation of CDKN2A and suppression of cyclin D1 gene expressions in malignant gliomas. J Exp Clin Cancer Res. 2011; 30:76.
17. Jiang YG, Peng Y, Koussougbo KS. Necroptosis: a novel therapeutic target for glioblastoma. Med Hypotheses. 2011; 76:350-352.

18. Liu B, Liu Y, Jiang Y. Podocalyxin promotes glioblastoma multiforme cell invasion and proliferation by inhibiting angiotensin-(1-7)/Mas signaling. Oncol Rep. 2015; 33:2583-2591.

19. Stupp R, Weber DC. The role of radio- and chemotherapy in glioblastoma. Onkologie. 2005; 28:315-317.

20. Kozak KR, Mahadevan A, Moody JS. Adult gliosarcoma: epidemiology, natural history, and factors associated with outcome. Neuro Oncol. 2009; 11:183-191.

21. Buckner JC, Shaw EG, Pugh SL, Chakravarti A, Gilbert MR, Barger GR, Coons S, Ricci P, Bullard D, Brown PD, Stelzer K, Brachman D, Suh JH, et al. Radiation plus Procarbazine, CCNU, and Vincristine in Low-Grade Glioma. N Engl J Med. 2016; 374:1344-1355.

22. Shahrokni A, Wu AJ, Carter J, Lichtman SM. Long-term Toxicity of Cancer Treatment in Older Patients. Clin Geriatr Med. 2016; 32:63-80.

23. Zheng Q, Yang L, Tan LM, Qin LX, Wang CY, Zhang HN. Stroke-like Migraine Attacks after Radiation Therapy Syndrome. Chin Med J (Engl). 2015; 128:2097-2101.

24. Biswas A, Kumar N, Kumar P, Vasishta RK, Gupta K, Sharma SC, Patel F, Mathuriya SN. Primary gliosarcoma-clinical experience from a regional cancer centre in north India. Br J Neurosurg. 2011; 25:723-729.

25. Zhang G, Huang S, Zhang J, Wu Z, Lin S, Wang Y. Clinical outcome of gliosarcoma compared with glioblastoma multiforme: a clinical study in Chinese patients. J Neurooncol. 2016; 127:355-362.

26. Salvati M, Caroli E, Raco A, Giangaspero F, Delfini R, Ferrante L. Gliosarcomas: analysis of 11 cases do two subtypes exist? J Neurooncol. 2005; 74:59-63.

27. Walker GV, Gilbert MR, Prabhu SS, Brown PD, McAleer MF. Temozolomide use in adult patients with gliosarcoma: an evolving clinical practice. J Neurooncol. 2013; 112:83-89.

28. Morantz RA, Feigin I, Ransohoff JR. Clinical and pathological study of 24 cases of gliosarcoma. J Neurosurg. 1976; 45:398-408.

29. Meis JM, Martz KL, Nelson JS. Mixed glioblastoma multiforme and sarcoma. A clinicopathologic study of 26 radiation therapy oncology group cases. Cancer. 1991; 67:2342-2349.

30. Parekh HC, O'Donovan DG, Sharma RR, Keogh AJ. Primary cerebral gliosarcoma: report of 17 cases. $\mathrm{Br}$ J Neurosurg. 1995; 9:171-178.

31. Perry JR, Ang LC, Bilbao JM, Muller PJ. Clinicopathologic features of primary and postirradiation cerebral gliosarcoma. Cancer. 1995; 75:2910-2918.

32. Sarkar C, Sharma MC, Sudha K, Gaikwad S, Varma A. A clinico-pathological study of 29 cases of gliosarcoma with special reference to two unique variants. Indian J Med Res. 1997; 106:229-235. 
33. Kumar N, Bhattacharyya T, Chanchalani K, Shalunke P, Radotra BD, Yadav BS. Impact of changing trends of treatment on outcome of cerebral gliosarcoma: A tertiary care centre experience. South Asian J Cancer. 2015; 4:15-17.

34. Rath GK, Sharma DN, Mallick S, Gandhi AK, Joshi NP, Haresh KP, Gupta S, Julka PK. Clinical outcome of patients with primary gliosarcoma treated with concomitant and adjuvant temozolomide: A single institutional analysis of 27 cases. Indian J Cancer. 2015; 52:599-603.

35. Singh G, Das KK, Sharma P, Guruprasad B, Jaiswal S, Mehrotra A, Srivastava AK, Sahu RN, Jaiswal AK, Behari S. Cerebral gliosarcoma: Analysis of 16 patients and review of literature. Asian J Neurosurg. 2015; 10:195-202.

36. Walker GV, Li J, Mahajan A, McAleer MF, de Groot JF, Azeem SS, Brown PD. Decreasing radiation therapy utilization in adult patients with glioblastoma multiforme: a population-based analysis. Cancer. 2012; 118:4538-4544.
37. Pretanvil JA, Salinas IQ, Piccioni DE. Glioblastoma in the elderly: treatment patterns and survival. CNS Oncol. 2017; 6:19-28.

38. Morgan ER, Norman A, Laing K, Seal MD. Treatment and outcomes for glioblastoma in elderly compared with non-elderly patients: a population-based study. Curr Oncol. 2017; 24:e92-e98.

39. Minniti G, Filippi AR, Osti MF, Ricardi U. Radiation therapy for older patients with brain tumors. Radiat Oncol. 2017; 12:101.

40. Arvold ND, Reardon DA. Treatment options and outcomes for glioblastoma in the elderly patient. Clin Interv Aging. 2014; 9:357-367.

41. Iwamoto FM, Cooper AR, Reiner AS, Nayak L, Abrey LE. Glioblastoma in the elderly: the Memorial Sloan-Kettering Cancer Center Experience (1997-2007). Cancer. 2009; 115:3758-3766. 